\title{
Quantification of Stress-induced Damage and Post-Fire Response of 5083 Aluminum
}

\section{Alloy}

\author{
Y. Chen ${ }^{\text {a }}$, S. B. Puplampu ${ }^{\text {c }}$, P.T. Summers ${ }^{\text {b }}$, B.Y. Lattimer ${ }^{\text {b }}$, D. Penumadu ${ }^{\text {c }}$, S.W. Case ${ }^{\text {a }}$ \\ ${ }^{a}$ Department of Engineering Science \& Mechanics, Virginia Tech, Blacksburg, VA 24061, USA \\ ${ }^{\mathrm{b}}$ Department of Mechanical Engineering, Virginia Tech, Blacksburg, VA 24061, USA \\ ${ }^{c}$ Department of Civil and Environmental Engineering, University of Tennessee, Knoxville, TN 37996, USA
}

\begin{abstract}
One of the major concerns regarding the use of lightweight materials in ship construction is the response of those materials to fire scenarios, including the residual structural performance after a fire event. This paper presents a study on creep damage evolution in 5083 marine-grade aluminum alloy and its impact on residual mechanical behavior. Tests conducted at $400^{\circ} \mathrm{C}$ and pre-selected tensile stress levels were interrupted at target amplitudes of accumulated engineering creep strains to investigate the stress-induced damage using ex-situ characterization. Two-dimensional optical and electron microscopy and three-dimensional X-ray tomography were utilized on samples extracted from these test specimens to characterize the external and internal creep damage. The stress-induced damage is primarily manifested as cavitation and dynamic microstructural evolution. Cavitation morphology, orientation and grain structure evolution were investigated on three perpendicular sample surfaces. A 3D examination of the damage state provided consistent damage information to that obtained from the 2D analysis. The post-fire mechanical properties were also evaluated and linked to the microstructural change. The competing processes of cavitation and grain structure evolution were investigated to develop an understanding of the stress-induced damage associated with high temperature creep.
\end{abstract}

Key words: Stress-induced damage; Cavitation; Grain elongation; Substructure strengthening; Residual strength

\section{Introduction}

Aluminum alloys are important for lightweight structural applications such as in aerospace and ship engineering. One potential concern with the use of aluminum is its behavior during extreme fire conditions. During fire exposure, material strength may be degraded and failure may occur because of the accumulation of fire damage. This damage to aluminum is complex and may be a combination of grain growth, precipitate cracking, and cavity formation [1-3]. In situations where the fire is extinguished and the structure remains intact, a key concern is the future performance of the structure. In order to predict this post-fire performance, a detailed understanding of the developed physical damage is necessary.

The time-temperature-stress material dependence, i.e., creep, is of major significance when considering material performance in fire. The incipient causes of creep failure include cavity nucleation, growth, and the subsequent coalescence of cavities within certain proximity. Cavity nucleation requires significant stress concentration that is orders of magnitude greater than the applied stress $[4,5]$. The early idea of vacancy agglomeration was from the nuclei (precipitation and solid inclusions) [6]. Grain boundary sliding and dislocation pile-up will generate sufficient local stresses and promote the formation of cavities, particularly when they occur against precipitates on the grain boundaries [7,8]. Existing cavities will also cause stress concentration and lead to new cavity formation as the damage progresses [9]. Once formed, cavity growth is primarily driven by two different mechanisms: vacancy diffusion and creep plasticity [10-12]. The comparison between diffusive cavity growth and plastic cavity growth demonstrated that cavities grow faster when driven by plasticity. Grain boundary sliding has also been considered as another mechanism for cavity growth [13].

A number of creep damage studies have been performed on superplastic aluminum alloys [14-17]. The effects of thermal exposure and stress state on the cavitation evolution were investigated. It was found that diffusive cavity growth and the effect of creep plasticity in the surrounding material can be coupled. Cavity growth was verified to be controlled by diffusion when cavities are small, then controlled by power-law creep when they are large [18]. Cavities may grow initially by diffusion, then by coupling of diffusion and creep plasticity, and finally by creep plasticity alone. A plastic cavity growth model was proposed, implying that with an initial cavity volume fraction value, cavities grow exponentially with the increase of creep strain [14].

Corresponding author. Tel.: +1 5405560907.

E-mail address: yanyun@vt.edu (Y. Chen). 
In addition to cavitation, grain size, shape, and texture have been found to evolve during the continuous creep deformation. Dynamic recovery and dynamic recrystallization also take place during high temperature creep deformation. Strengthening mechanism based on grain structure change has been studied: The Hall-Petch relation established an effective method to estimate material strength caused by average grain size change [19]. The effect of grain shape change on the material strength has also been studied [20]. Grain aspect ratio evolution has been proposed to cause the material yield strength change. Yield strength will increase as structure evolves from an equiaxed grain structure to high aspect ratio grains. A model coupling grain orientation and grain size/shape effect has been developed [21]. Based on the various strengthening mechanisms in AlMg alloy including grain boundary strengthening, solute strengthening, precipitate hardening, dislocation wall strengthening, dislocation sub-structure was found to be the dominant strengthening mechanism in Al5083 [22]. The grain structure strengthening effects can be also linked to the subgrain structure strengthening mechanism. Saturated dislocations have been generated in the as-received Al5083 during the strain hardening process. Dynamic recovery process associated with the increase in grain aspect ratio will cause refinement of the dislocation sub-structures. Smaller subdomains will be developed and result in strengthening in the residual material strength [23].

Despite these efforts, a lack of understanding still exists on the effect of creep cavity growth and dynamic microstructure evolution on the residual material strength for the 5083 aluminum alloy after fire exposure. This work seeks to establish a fundamental level of damage quantification to aid in the analysis of these simultaneously evolving mechanisms. In this paper, a uniaxial stress state was induced during tensile creep experiments to simulate fire exposure conditions. Damage associated with the creep response was examined by two-dimensional techniques including high resolution optical microscopy, SEM and TEM, and three-dimensional X-ray micro-tomography. The cavitation and dynamic microstructure evolution in 5083H116 aluminum alloy during creep were examined to study the damage mechanism. Residual mechanical properties were also determined to evaluate the post-fire performance of the material and its dependence on the stress state during prior elevated temperature exposure. The competing process between cavitation and grain structure evolution was investigated and correlated to the residual material strength.

\section{Materials and Sample Geometry}

For this work, the marine-grade aluminum alloy 5083-H116 is investigated. Aluminum 5083-H116 is a work-hardened alloy that contains $4-4.9 \%$ of magnesium as its major alloying element. The nominal chemical composition is shown in Table 1. This alloy is known for exceptional performance in extreme environments and its excellent resistance to seawater and corrosive chemicals. Thus, it is frequently used in marine applications. The material was supplied by Alcoa in the form of $6.35 \mathrm{~mm}$ thick sheets.

Table 1 Chemical composition of Al 5083 material (wt.\%)

\begin{tabular}{|c|c|c|c|c|c|c|c|c|c|}
\hline $\begin{array}{c}\text { Chemical } \\
\text { element }\end{array}$ & $\mathrm{Al}$ & $\mathrm{Mg}$ & $\mathrm{Mn}$ & $\mathrm{Si}$ & $\mathrm{Fe}$ & $\mathrm{Zn}$ & $\mathrm{Ti}$ & $\mathrm{Cr}$ & $\mathrm{Cu}$ \\
\hline Al5083 & Balance & $4.0-4.9$ & $0.4-1.0$ & 0.4 & 0.4 & 0.25 & 0.1 & $0.05-0.25$ & 0.1 \\
\hline
\end{tabular}

Test specimen geometry was selected based on the ASTM standard E21-09, as illustrated in Fig. 1. Specimens were machined from the as-received plate to dog-bone geometry with the longitudinal axis oriented in the rolling direction. The specimens had an overall length of $304.8 \mathrm{~mm}$ and uniform thickness of $6.35 \mathrm{~mm}$, with a gage length and width of 190.5 and $12.7 \mathrm{~mm}$, respectively.
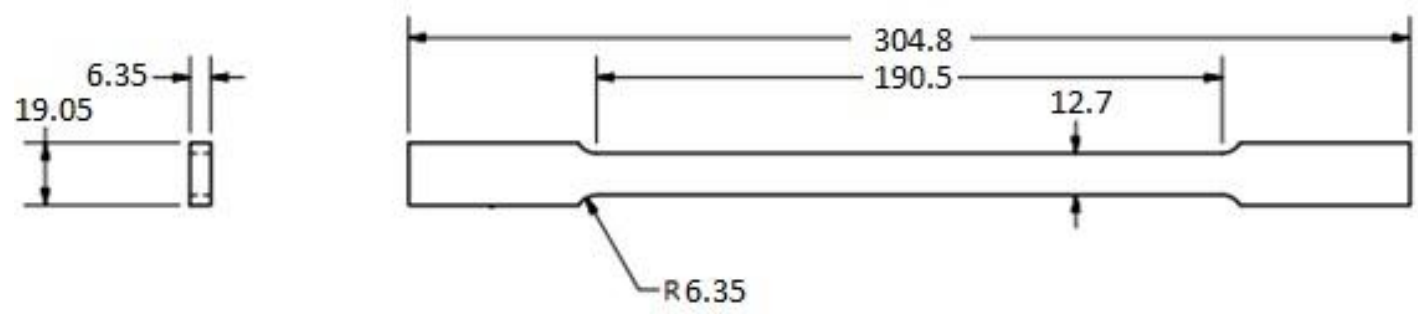

Fig. 1 Tensile test specimen geometry (mm) 


\section{Experimental Methods}

\subsection{Simulated Thermal-Mechanical Tests}

To simulate the thermal-mechanical loading condition and induce creep damage to the material, specified temperatures and stress levels were selected to perform creep tests. Previous work on the $\mathrm{Al} 15083$ alloy revealed that $400^{\circ} \mathrm{C}$ was a critical temperature at which grain recrystallization was completed, and the lowest post-fire yield and ultimate strengths occurred after $400^{\circ} \mathrm{C}$ exposure [24]. Thus, $400^{\circ} \mathrm{C}$ was selected to study creep damage. All creep tests were performed using an MTS 880 Test Frame and an Ameritherm 5060LI induction heater. A Micro-Epsilon optical pyrometer was used to provide temperature measurements for the feedback control of the sample temperature via the induction heater. Samples were heated from room temperature to $400^{\circ} \mathrm{C}$ at a controlled heating rate of $50^{\circ} \mathrm{C} / \mathrm{min}$. A pre-selected engineering tensile stress of $17 \mathrm{MPa}$, $15 \mathrm{MPa}$, or $14 \mathrm{MPa}$ was applied once the sample reached $400^{\circ} \mathrm{C}$. Samples were then held at a constant load and temperature to achieve the desired creep strain. Engineering creep strains were measured over a $22 \mathrm{~mm}$ gage length using a Fiedler Optoelektronik GmbH Laser Extensometer, as shown in Fig. 2.

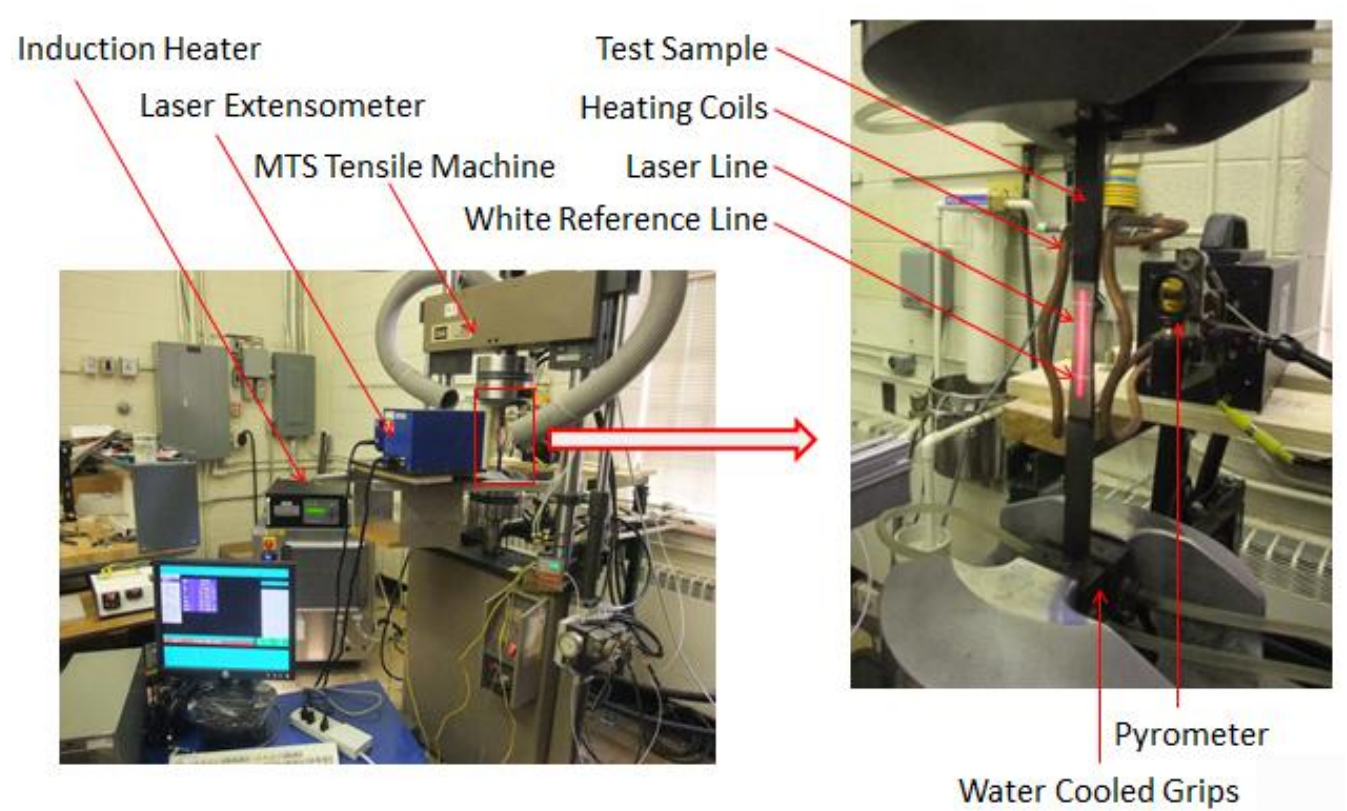

Fig. 2 Simulated fire-load conditioning test set up

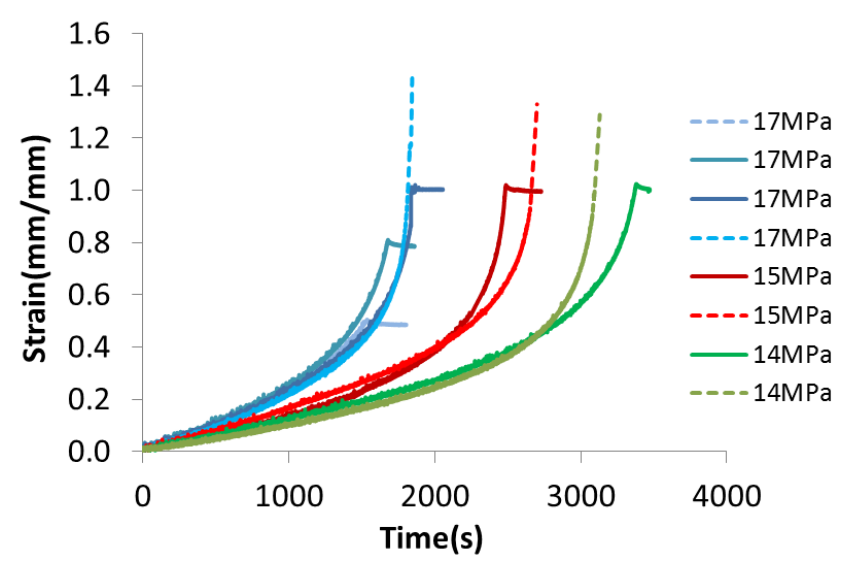

Fig. 3 Creep curves of the fire-load conditioning tests for Al5083 samples 
As shown in Fig. 3, the creep tests were interrupted/stopped at engineering strains of $50 \%, 80 \%, 100 \%$, and $143 \%$ for samples subjected to a constant tensile stress of $17 \mathrm{MPa} ; 100 \%$ and $133 \%$ axial strain for $15 \mathrm{MPa}$; and $100 \%$ and $144 \%$ for 14MPa. As expected, samples demonstrated necking in the center of the gauge region, indicating the presence of creepinduced damage. Sections were cut from the necked regions (approximately $6 \mathrm{~mm}$ in length) to examine creep damage. Characterization was performed using two-dimensional and three-dimensional techniques on all three perpendicular sample surfaces (rolling, transverse and normal surfaces as shown in Fig. 4) to examine the cavity morphology and grain structure. Multiple creep samples stressed to identical strains were prepared and tensile tests at ambient conditions were performed to measure residual mechanical properties. To link the microstructural details to post-fire residual mechanical properties, residual material strength is calculated based on the deformed cross section area after the creep experiment has been completed.

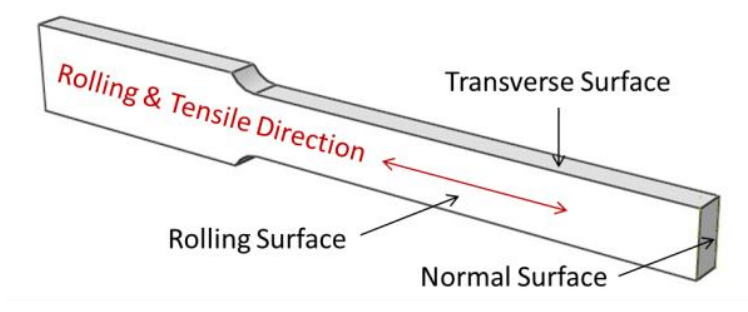

Fig. 4. Sectioning terminology

\subsection{Two-Dimensional Microstructure Analysis}

Two-dimensional damage quantification was performed by high resolution microscopy, FEI Quanta 600 FEG SEM and Philips EM420 TEM. To study the cavitation evolution, damage samples were well polished to a mirror finish and examined by the secondary electron detector of SEM at a low accelerating voltage. SEM images for each sample, including ten locations for each surface (rolling, transverse and normal), were collected to quantify the creep damage. Images taken at 500x magnifications were selected to study cavity evolution. This magnification shows a sample surface area of $512 \times 443 \mu \mathrm{m}$, which is the optimal size to analyze cavities larger than $1 \mu \mathrm{m}$ and measure the overall creep damage state. To estimate the cavity volume fraction, grey-scale SEM images were converted to binary images using a thresholding process in Matlab. Suitable threshold value was adjusted for each SEM images based on their brightness. The average cavity size was calculated based on the total cavity area and the number of cavities in each image (assuming spherical cavities and circular crosssections).

Polished samples were then etched by Barker's solution and examined by high resolution microscopy to investigate dynamic microstructural evolution. Grain dimensions were calculated using line intersection method from each direction. Grain size was calculated by averaging the grain length, width and height on all three material planes, while grain aspect ratio was estimated by the grain length and width on the rolling material plane.

Duplicated damaged samples were sectioned to approximate $1.5 \times 1.5 \times 1 \mathrm{~mm}$, and then mirror polished both sides down to about $20 \mu \mathrm{m}$ thick. The $20 \mu \mathrm{m}$ polished sample were glued to a 3mm-diameter circular copper grid and iron milled for 3-5 hours to generate thin area for TEM observation. The dislocation and substructure evolution at low and high strained materials were examined by Philips EM420 TEM.

\subsection{Three-Dimensional X-ray Tomography}

Three dimensional damage analyses were performed by Siemens microCAT in vitro system and with an exposure time of $8500 \mathrm{~ms}$ per radiograph. The $\mathrm{xCT}$ scans at $130 \mathrm{kV}$ were acquired for progressive strain levels at resolutions ranging from 5 to $6 \mu \mathrm{m} /$ pixel. As a reference, an xCT scan of the A15083 as-received sample was acquired at $7 \mu \mathrm{m} / \mathrm{pixel}$. Details for the scans are reported in Table 2. 
Table 2 XCT scans details for Al5083 samples

\begin{tabular}{|c|c|c|c|c|}
\hline Strain (\%) & Stress (MPa) & Rotation steps & $\begin{array}{c}\text { Source to object } \\
\text { distance }(\mathrm{mm})\end{array}$ & Voxel size \\
\hline $0 \%$ & 0 & 2800 & 91.44 & 6.98 \\
\hline $100 \%$ & 15 & 3000 & 65.67 & 5.01 \\
\hline $133 \%$ & 15 & 3000 & 78.38 & 5.98 \\
\hline $143 \%$ & 14 & 3000 & 71.84 & 5.46 \\
\hline
\end{tabular}

Image acquisition was followed by cross-section reconstruction using Octopus [25]. Projections were filtered and normalized. "Bad" pixels on the detector can introduce spurious features in the reconstruction by constantly appearing in each successive projection. Images were de-speckled to reduce formation of these artifacts. Projections were normalized using open beam and dark field images; this procedure also accounts for beam flux variations throughout the duration of the scan. Normalized projections were then converted to sinograms, which represent intensity variations of one detector horizontal line as the sample is rotated through $360^{\circ}$. Sinograms are images whose width coincides with the horizontal number of detector pixels and whose height is determined by the number of projections. Reconstructed cross-section images were obtained from the sinograms.

Particle analysis using ImageJ is effective for total cavity fraction calculation and cavity size distribution in two dimensions. To better understand cavity shape and distribution throughout the samples, Avizo ${ }^{\circledR}$ was employed for 3D visualization and analysis. 3D visualization of the reconstructed cross-section images allows qualitative and quantitative analysis of features of interest such as surface area, volumetric and spatial distribution measurements.

\section{Results and Discussion}

\subsection{Damaged Samples}

As mentioned previously, samples were interrupted at various creep strains to isolate various stress-induced damage states, as shown in Fig. 5. Both unfractured and fractured (broken) samples tested at 17MPa, 15MPa and 14MPa are shown in Fig. 5a-c with the fractured samples on the right side of each sample set. Large plastic deformation and severe necking were observed in the fractured samples. The fracture surfaces are almost necked to a thin line, with a $90 \%$ reduction in area, indicating highly ductile failure. Fig. 5d shows an isolated view of the necked portion for a highly deformed and damaged sample. Several cracks are observed on the rolling surface indicating impending failure. Similar cracks are observed for the $17 \mathrm{MPa}$, $143 \%$ creep strain sample. These highly damaged samples have similar elongation to the fracture ones shown in the right in Fig. 5 indicating they have reached a state just prior to material failure. For the $15 \mathrm{MPa}$ and $133 \%$ strain sample, necking is not as severe as those strained to $143 \%$ and $144 \%$; no cracking was observed on the sample surface, implying that this sample was approaching failure but had not yet reached the just-before-fracture state. 


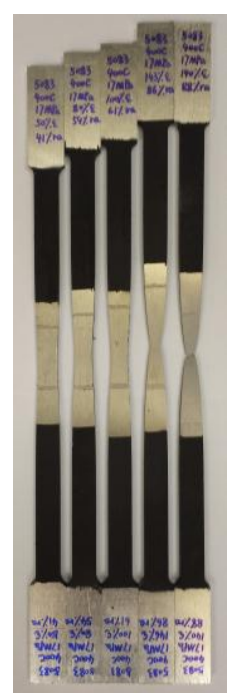

(a)

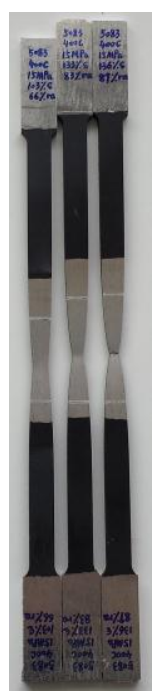

(b)

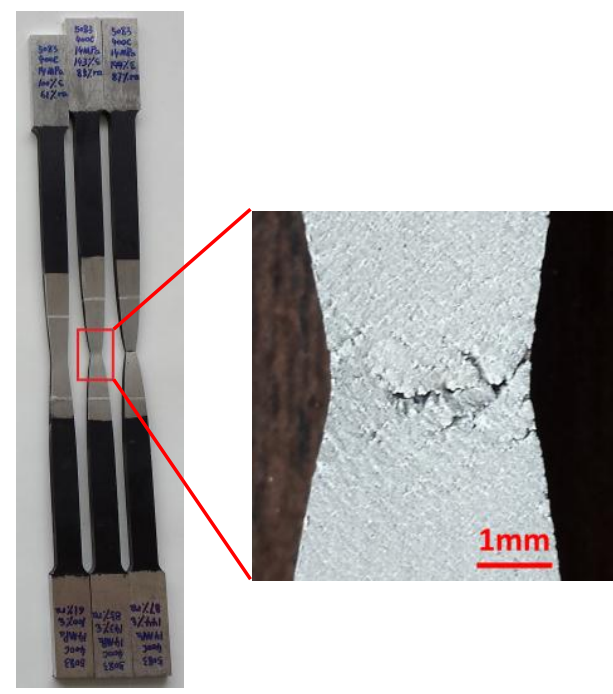

(c) (d)

Fig. 5 (a) $17 \mathrm{MPa}, 50 \%, 80 \%, 100 \%, 143 \%$ creep strain and fracture samples, (b) $15 \mathrm{MPa}, 100 \%, 133 \%$ creep strain and fracture samples, (c)14MPa, 100\%, 144\% creep strain and fracture samples, and (d) Necking region of the just-beforefracture sample

\subsection{Two-Dimensional Damage Quantification}

\subsubsection{Cavitation Evolution}

Creep damage is manifested as the cavitation evolution with the development of creep strain, which can be represented as the increase in the number of visible cavities as well as the growth of those cavities. Fig. 6a shows the as-received microstructure, no cavities were observed in the aluminum matrix except for several randomly distributed $\mathrm{Fe}$ and $\mathrm{Mn}$-rich intermetallic inclusions, which appear darker on the light aluminum background. Fig. 6b-e displays damaged microstructures at various strain levels examined by SEM. In thermo-mechanical test conditions, significant stress concentrations will be generated around the intermetallic inclusions and on the junction of grain boundaries. Cavities will nucleate at these locations due to sufficient local stress concentrations during the plastic deformation.

Strain localization and necking occurs with the increasing plastic strain. Severe necking coincides with stress-induced damage accumulation in the sample. For creep microstructures at strains lower than $100 \%$, the cavities appear mostly spherical in shape and randomly distribute on the microstructure. The cavity sizes at these strains are relatively small and similar. As creep strain exceeds 100\%, the cavities begin to grow and agglomerate; more cavities in elongated shape appear in the microstructure. This indicates the cavities are stretched and coalesce with the accumulation of creep strain. After the sample deforms beyond $100 \%$ creep strain and approaches failure, more spherical cavities were stretched and coalesce into large cylindrical cavities. These crack-like cavities are parallel to the rolling/tensile direction with lengths ranging from $50 \mu \mathrm{m}-150 \mu \mathrm{m}$ and aspect ratios greater than 5 . 


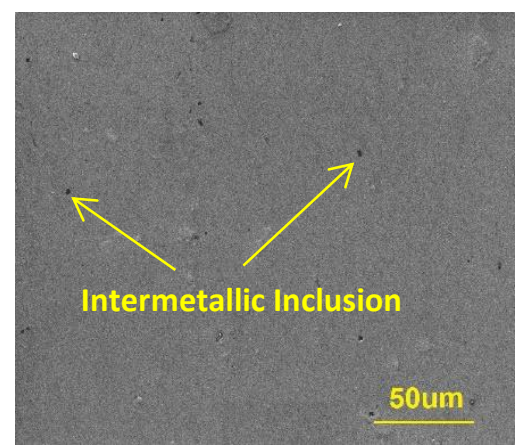

(a)

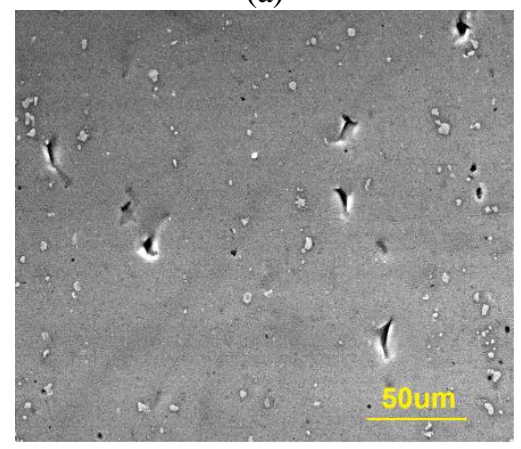

(d)

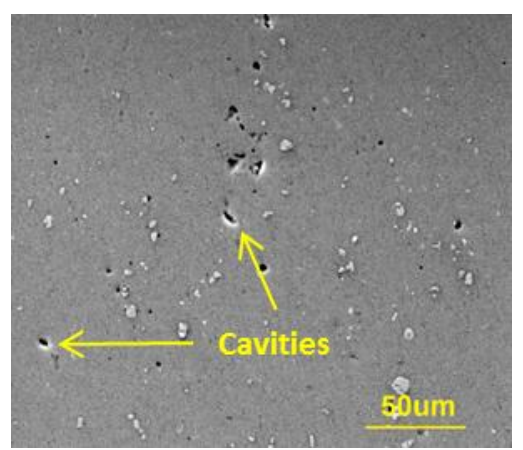

(b)

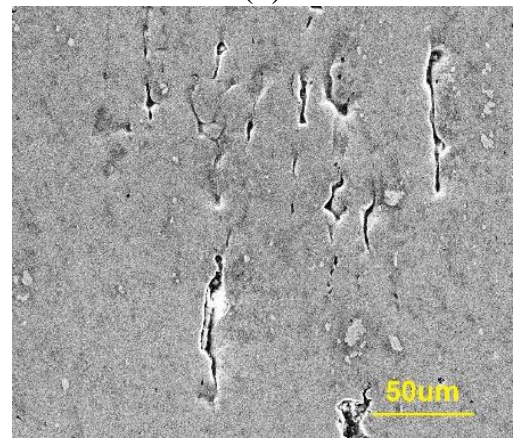

(e)

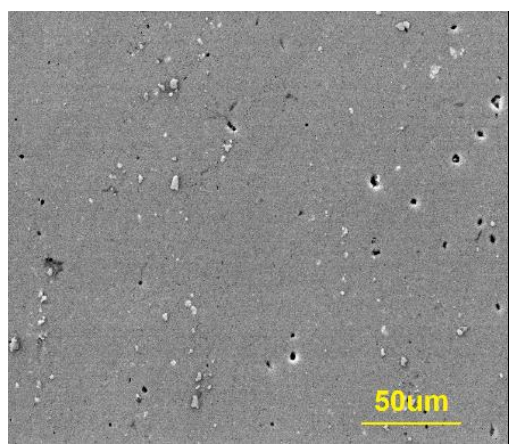

(c)

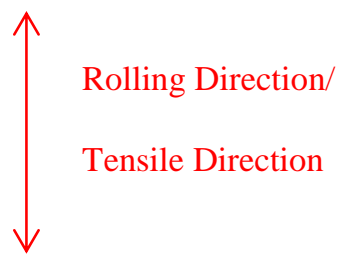

Fig. 6 Cavitation evolution with creep strain (SEM, 500x, rolling surface) (a) as-received $\varepsilon=0 \%$; and $400^{\circ} \mathrm{C} 17 \mathrm{MPa}$ applied stress (b) $\varepsilon=50 \%$, (c) $\varepsilon=80 \%$, (d) $\varepsilon=100 \%$, and (e) $\varepsilon=143 \%$

Closer inspection of the microstructures on each sample surface gives more evidence of creep cavity orientation, as shown in Fig. 7. The rolling and transverse material planes display vertical crack-like cylindrical cavities along the rolling/tensile direction, while spherical cavities are randomly distributed on the normal material plane. The cavity morphologies indicate that during large plastic deformation, creep cavities grew from an initial spherical shape into the longer, cylindrical shapes along the rolling/tensile direction. Similar cavity morphologies are displayed on all three material planes of the $17 \mathrm{MPa}$ and 14MPa samples, indicating similar damage has been developed during the creep tests within 14MPa-17MPa range. Several small cracks are also observed in the microstructure just prior to rupture, as shown in Fig. 7f. These cracks grow transversely to the rolling/tensile direction and connect the closely spaced cavities; implying micro-cracks will be developed after cavity coalescence and crack propagation causes eventual sample failure.

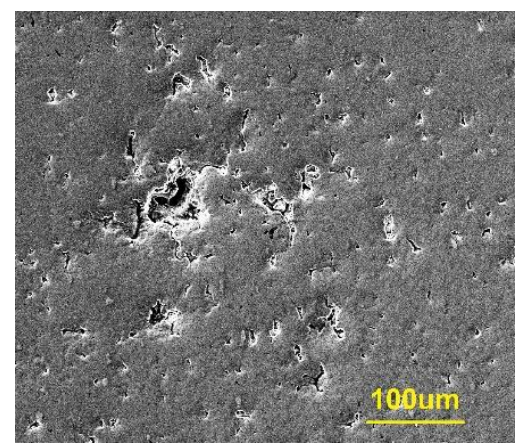

(a)

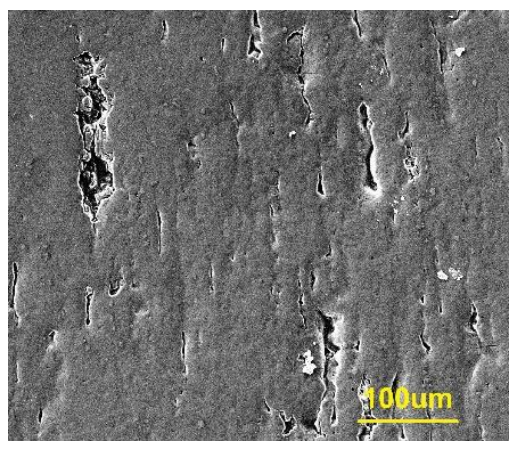

(b)

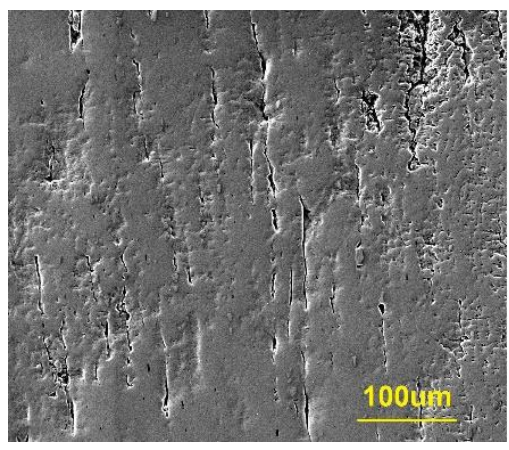

(c) 


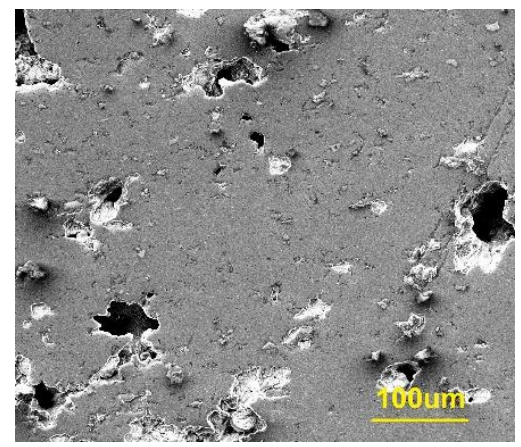

(d)

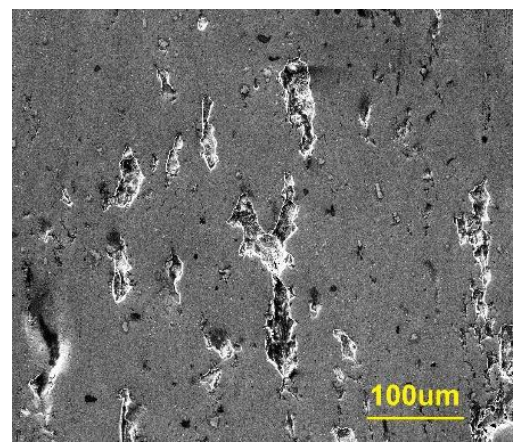

(e)

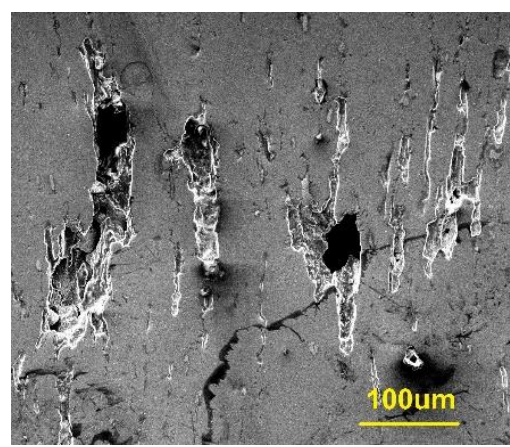

(f)

Fig. 7 Just-before-rupture samples creep cavity morphology on each material plane (SEM, 500x) (a)17MPa, Normal, (b)17MPa, Rolling, (c)17MPa, Transverse, (d)14MPa, Normal, (e)14MPa, Rolling, and (f)14MPa, Transverse

Cavitation can be quantified based on cavity volume fraction and cavity size in the deformed material. As shown in Fig. 8, the cavitation parameters for the above microstructures are summarized for multiple creep strain and stress levels. The standard deviations during image analysis are also shown to suitably give a quantitative sense on possible ranges in interpreted porosity. For samples subjected to $17 \mathrm{MPa}$ stress, the cavity volume fraction shows a gradually increasing trend with increasing creep strain below $100 \%$; however, it is limited to $1 \%$ cavity volume fraction at $100 \%$ strain. As material continues to deform above $100 \%$ creep strain, localized necking becomes more severe and the rate of cavity growth dramatically increases. However, the critical cavity volume fraction before sample failure is still relatively small (approximately $4.7 \%$ for $17 \mathrm{MPa}$ strained sample).

Similar quantification was performed for samples with $14 \mathrm{MPa}$ and $15 \mathrm{MPa}$ applied stress. The $100 \%$ creep strained samples and those at the just-before-fracture state (approximately $140 \%$ strain) were selected to compare the stress-dependence of creep damage. As shown in Fig. 8a, the cavity volume fractions at 100\% strain are about 1\% for all 14-17MPa creep samples. The just-before-fracture data of 14MPa and 17MPa samples also gives similar cavity volume fraction (around 5\%), which corresponds to the similar microstructural damage states in Fig. 7. Thus 5\% cavitation can be defined as the critical cavity volume fraction before sample failure. The $15 \mathrm{MPa}, 133 \%$ strain sample exhibits about $3 \%$ cavity volume fraction, which lies between the volume fractions at $100 \%$ axial strain and the just-before-rupture state. The standard deviations of the cavity volume fraction at multiple locations are more than $+/-1 \%$ for the just-before-fracture state, which is quite large compared to the small standard deviations below $100 \%$ strain. These large values of standard deviation associated with cavity volume fraction are also coincident with the non-uniform distribution of large crack-like cylindrical cavities inside the necking region when material is approaching failure.

Cavity size evolution with creep strain is similar to that for cavity volume fraction. As shown in Fig. 8 b, the average cavity size gradually increases below $100 \%$ strain and sharply increases when creep strain exceeds $100 \%$. Cavity sizes are similar at the same creep strain levels for all 14-17MPa applied stress levels. The random distribution of large crack-like cavities also causes larger standard deviation of cavity size at the just-before-fracture states.

Previous work has indicated that the cavity growth translated from diffusion-controlled to plasticity-controlled with the enlargement of cavity size. In the small cavity state, cavity growth is dominated by the diffusion-controlled mechanism and migrates along the boundaries. In contrast, plasticity-controlled growth is the dominant mechanism at large cavity sizes under high creep strain [26]. The superplastic cavity growth model [27] suggests a critical cavity size of $0.5 \mu \mathrm{m}$. When average cavity size is smaller than $0.5 \mu \mathrm{m}$, cavity growth is diffusion-controlled; cavitation is plasticity-controlled when the cavity size is greater $0.5 \mu \mathrm{m}$. Fig. $8 \mathrm{~b}$ shows that the average cavity sizes are greater than $0.5 \mu \mathrm{m}$ for all tested conditions. Thus, cavity growth during Al5083 creep is primarily plasticity-controlled based on the data gathered in this work.

When cavity growth is plasticity-controlled, the cavity volume fraction can be given by a simple model in Eq. (1)[28]:

$$
\phi=\phi_{0} \exp (\eta \varepsilon)
$$


Where $\phi$ is the cavity volume fraction at the strain $\varepsilon, \phi_{0}$ is the initial cavity volume fraction of the as-received material, and $\eta$ is a growth parameter usually ranging from 2 to 4 , and is dependent on material, strain rate, temperature, and grain size. An exponential fit based on the data collected at all stress levels in Fig. 8a yields an $\eta$ of 2.07, which lies between the upper bound value of 2.5 calculated by Cocks and Ashby's model and the conservative value of 1 predicted by Budiansky [29, 30].

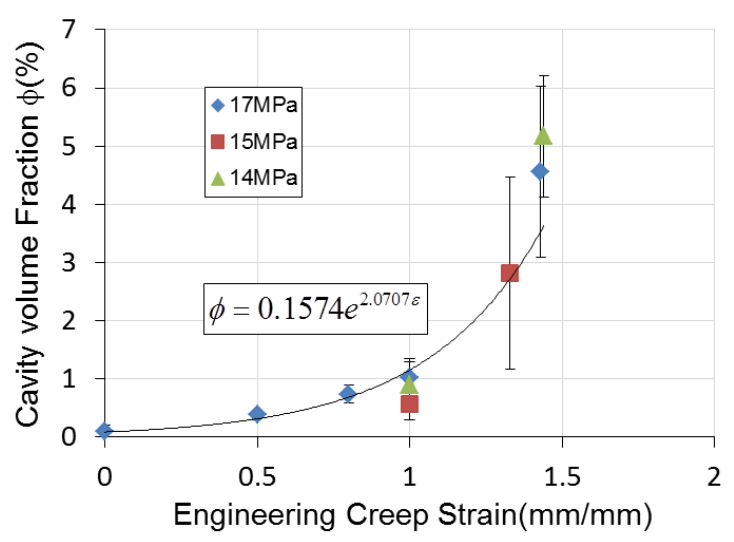

(a)

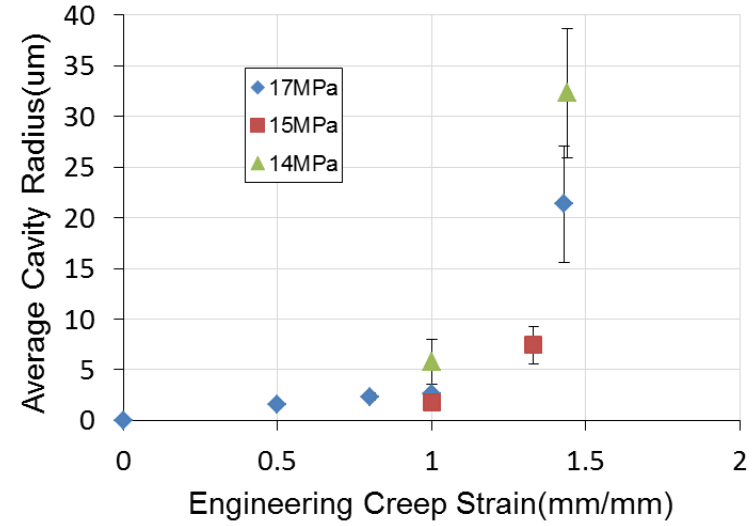

(b)

Fig. 8 (a) Cavity volume fraction versus creep strain, (b) Average cavity size evolution versus creep strain

\subsubsection{Dynamic Microstructure Evolution}

In addition to cavitation, another feature of stress-induced creep damage is dynamic microstructure evolution with the accumulation of creep strain. The as-received state is shown in Fig. 9a, in which large pancake grains oriented with the rolling direction are observed on the rolling surface. The as-received average grain size is approximately $80 \mu \mathrm{m}$ by averaging grain length, width and height on all three material planes. Some cavities observed inside the grains are due to the precipitates removed by the etchant. After non-stressed $400^{\circ} \mathrm{C}$ exposure, static grain recrystallization process is completed; the original pancake grain structure has evolved into an equiaxed grain structure with the average grain size decreasing to $46 \mu \mathrm{m}$, as shown in Fig. 9b. Grain aspect ratio also evolved from 2.81 to 0.91 in the rolling surface after static grain recrystallization.

The dynamic microstructure evolution during $400^{\circ} \mathrm{C}$ creep process is illustrated in Fig. 9c-f, in which materials are strained to various strain levels. With the accumulation of mechanical strain, the equiaxed grain structure is increasingly stretched to the thin, long grain structure along the rolling/tensile direction. Grain aspect ratio calculated in Fig. 10a increases from 0.91 at $0 \%$ mechanical strain to 7.5 at $143 \%$ creep strain, which indicates that grains are highly elongated along the tensile direction to accommodate large plastic deformation during creep. When grain boundaries become serrated in the highly elongated grains and are only separated by small distance, geometric dynamic recrystallization process will take place [31]. As shown in Fig. 9f, during creep deformation grains are increasingly flattened till the before rapture state. Grain boundaries become serrated and separated by only a small distance, in some locations small new grains are observed to nucleate due to the contact of the serrated boundaries.

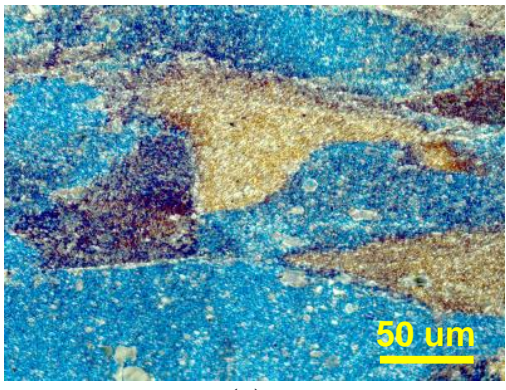

(a)

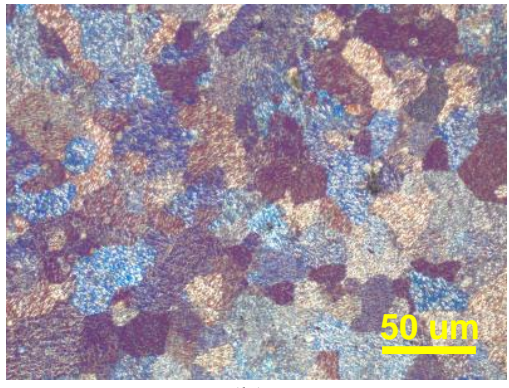

(b)

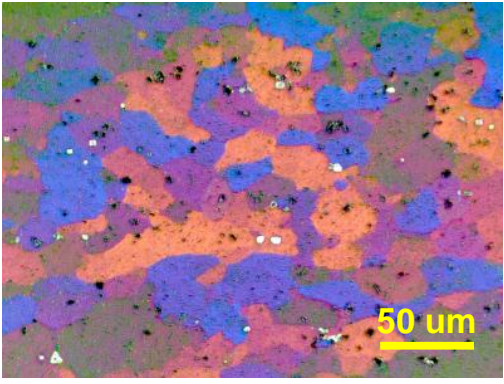

(c) 


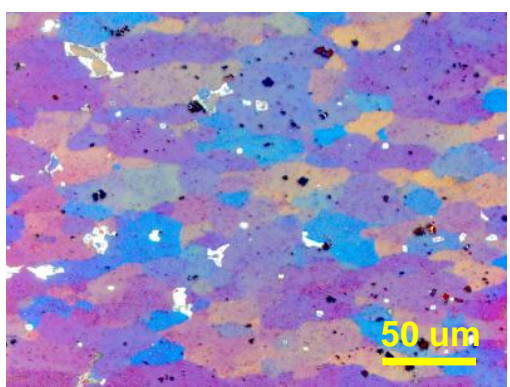

(d)

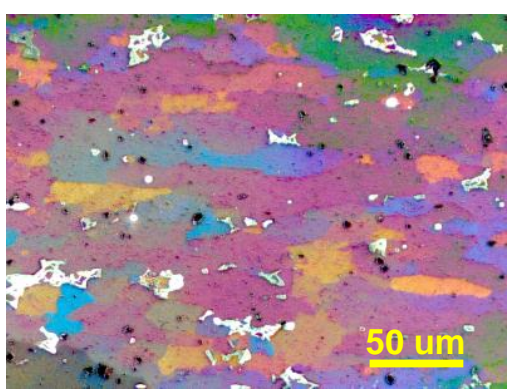

(e)

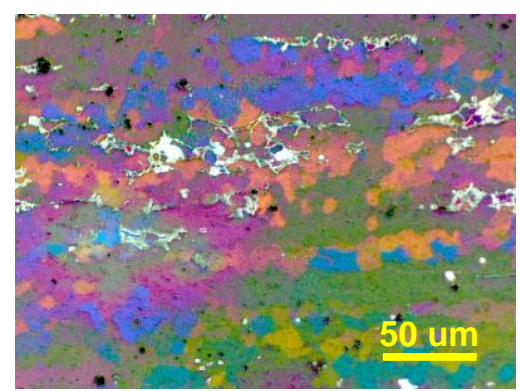

(f)

Rolling / Tensile Direction

Fig. 9 Grain structures (560x, rolling surface) of (a) as-received sample, (b) $400^{\circ} \mathrm{C}, 0 \mathrm{MPa}, \varepsilon=0 \%$, and (c)-(f) $400^{\circ} \mathrm{C}, 17 \mathrm{MPa} \mathrm{creep}$ samples, $\varepsilon=50 \%, 80 \%, 100 \%$ and $143 \%$

To evaluate the contribution of grain deformation to creep process, the relation between longitudinal grain strain and sample true strain are listed in Fig. 10b. The longitudinal grain strain is estimated based on the longitudinal grain deformation. The strained grain length in Fig. 9c-f divided by the equiaxed grain length on the tensile direction in Fig. 9b gives the longitudinal grain strain for strained samples. True creep strain in the localized neck region is calculated from the reduced cross sectional area data, which is based on the isochoric deformation concept.

As shown in Fig. 10b, the longitudinal grain strain exhibits similar value with true strain in the neck region at the low creep strain levels. However, the discrepancy between longitudinal grain strains and the localized true strain increases progressively with the accumulation of creep strain. At the just-before-fracture state, only about $60 \%$ of the localized true strain is contributed by the longitudinal grain strain. This observation reveals that grain deformation in A15083 can accommodate the small creep strains, but shows high incompatibility with larger creep strains. Grain boundary sliding will take place when the grain deformation cannot accommodate the large plastic deformation and will contribute to the creep strain. The quantitative data in Fig. 10b indicates that more than 30\% of localized true strain is contributed by grain boundary sliding before Al5083 creep failure. Large standard deviation associates with before fracture data due to new grains formation at the stage before creep failure.

Only small spherical cavities are observed to nucleate at the grain boundaries at low creep strains (50\% strain). The formation of these cavities is promoted by stress concentrations at the grain boundaries, particularly at grain triple junctions and where precipitates are located on grain boundaries. As the sample creeps to higher strain levels ( $80 \%$ and $100 \%$ strain), cavity growth is controlled by creep plasticity; large creep strains are produced around the surface of the cavities and small cavities are driven to coalesce into larger ones [14]. Grain boundary sliding occurs during this plastic creep process to cause significant stress concentration in the plane of the sliding boundary which also promotes the cavity growth [13]. During this stage, small spherical cavities are developed into larger crack-like cylindrical cavities along the grain boundaries. 


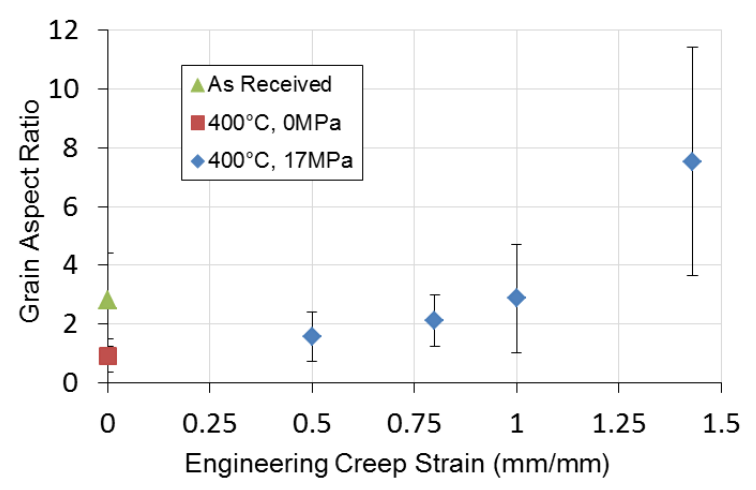

(a)

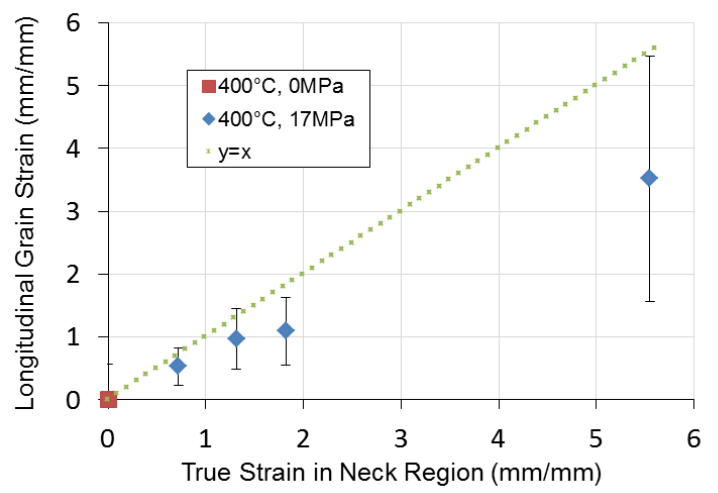

(b)

Fig. 10 (a) Grain aspect ratio (calculated based on rolling surface grains) evolution with engineering creep strain, (b) Longitude grain strain versus true strain in neck region (converted based on area of reduction in the neck)

The just-before-rupture state in Fig. 9f shows a clear view of cavity growth and coalescence at high creep strains. Grain boundaries are clearly identified on the imaged sample surface. Some closely spaced cavities coalesce during the grain boundary sliding. Crack-like cylindrical cavities are developed between grains and grow along the grain boundaries. Cavity coalescence is the final stage of the cavity growth, creep plasticity-controlled mechanism is an important factor in this stage [32]. In some cases, fracture occurs when these intergranular cavities disintegrate the grains between them and coalesce across grains to develop into the transgranular cavities. However, intergranular cavity coalescence is distributed quite heterogeneously on the grain boundaries. Some boundaries have reached a state of coalescence while others have not yet begun. As a consequence, some grain boundary facets may be separated before fracture occurs due to the stress being redistributed during heterogeneous cavitation. With continuous plastic deformation, the separated grain boundaries will be connected and microcracks are then developed to connect the closely spaced cavities in the just-before-fracture state, as show in Fig. $7 \mathrm{f}$ in section 4.2.1. The interconnection and propagation of these microcracks will lead to fracture of the material.

\subsection{Three-Dimensional Damage Quantification}

Two-dimensional surface analysis is limited to observable damage on the imaged sample surface, while three-dimensional Xray tomography provides a volumetric representation of the internal stress-induced cavitation damage. Raw tomography data is reconstructed to obtain cross section images showing the internal features of the samples. The reconstructed cross-section images for 100\%, 133\% and 143\% creep strain samples are shown in Fig. 11a, d and g. With the intent of quantifying cavity volume fraction, a region of interest (ROI) was selected for each sample through a number of reconstructed cross section slides thus creating a sub-volume, as shown in Fig. 11b, e and h. The sub-volume for cavity fraction analysis for each sample was selected to be completely embedded in the sample. Knowing the image resolution in microns per pixel, dimensions can be easily converted from pixels to microns. Thresholding based image segmentation was carried out on the sub-volume to make use of the "Particle Analysis" feature in ImageJ. In the thresholding process, images are converted to binary black and white images with cavities showing in white against a black background, as shown in Fig. 11c, f and g. Binarized images were median filtered to eliminate noise and smooth cavity edges.

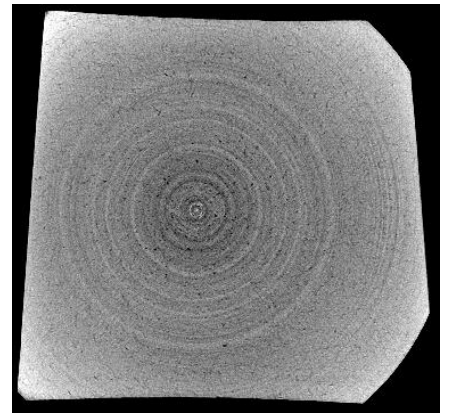

(a)

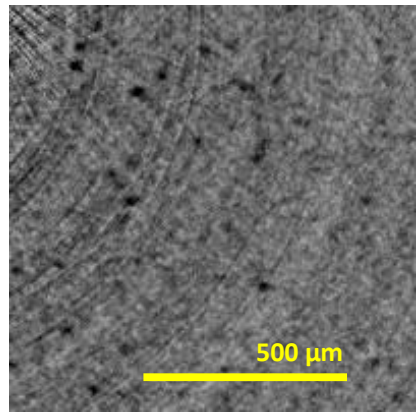

(b)

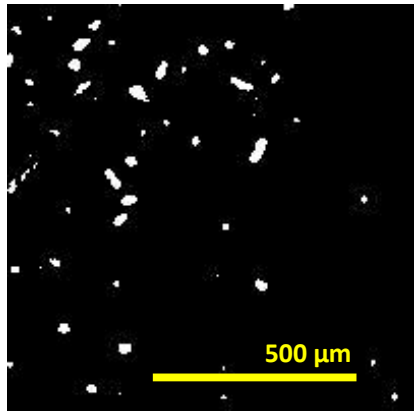

(c) 


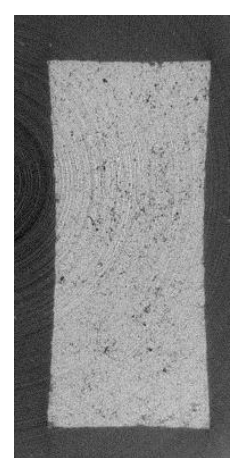

(d)

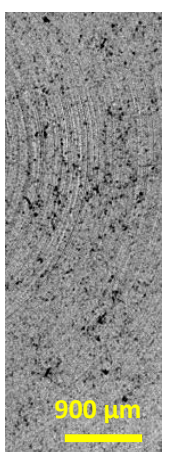

(e)

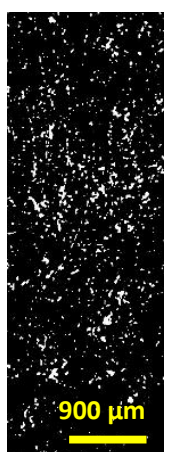

(f)

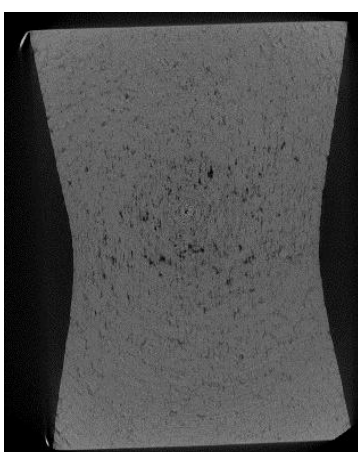

(g)

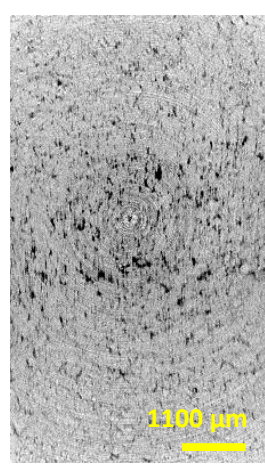

(h)

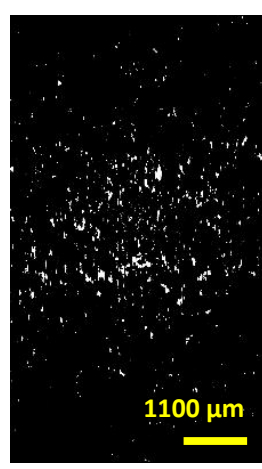

(i)

Fig. 11 15MPa, 100\% strain (a) reconstructed cross section, (b) reconstructed ROI, (c) bitmap ROI

$15 \mathrm{MPa}, 133 \%$ strain (d) reconstructed cross section, (e) reconstructed ROI, (f) bitmap ROI

$14 \mathrm{MPa}, 143 \%$ strain $(\mathrm{g})$ reconstructed cross section, (h) reconstructed ROI, (i) bitmap ROI

During particle analysis, each cavity is identified and labeled with an associated volume. Summing up the cavity volume and dividing by the total volume analyzed, the cavity volume fraction value is obtained for each sub-volume studied. Both threedimensional and two-dimensional analysis results are presented in Table 3. The cavity volume fraction data analyzed by 3-D X-ray tomography are consistent with the 2-D SEM results, showing dramatic increase with creep strain increase above 100\% strain. Compared to the $15 \mathrm{MPa} 100 \%$ strain sample, the cavity volume fraction increase by a factor of 10 in the just-beforerupture sample (143\% creep strain). However, 3-D results are relatively conservative compared with the 2-D SEM analysis. This may be due to the restriction of $\mathrm{xCT}$ scanning resolution $(5 \mu \mathrm{m} / \mathrm{pixel})$, under which the cavities smaller than $5 \mu \mathrm{m}$ cannot be identified.

Table 3: Cavity fraction analysis for Al5083 creep samples

\begin{tabular}{|c|c|c|}
\hline Cavity volume fraction (\%) & 3D X-ray Tomography & 2D SEM \\
\hline $15 \mathrm{MPa}, 100 \%$ strain & $0.34 \pm 0.06 \%$ & $0.56 \pm 0.25 \%$ \\
\hline $15 \mathrm{MPa}, 133 \%$ strain & $2.95 \pm 0.10 \%$ & $2.81 \pm 1.65 \%$ \\
\hline $14 \mathrm{MPa}, 143 \%$ strain & $3.10 \pm 0.12 \%$ & $5.17 \pm 1.04 \%$ \\
\hline
\end{tabular}

Subsequent to 2-D analysis, the cross-section images from each sub-volume were imported to Avizo 3D for 3-D visualization. Similar to 2-D analysis, the volume rendered image requires thresholding to facilitate particle identification and analysis. Identified cavities can then be categorized based on various parameters such as volume, surface area, and barycenter position.

Fig. 12 shows one of the 3D reconstructed samples and the internal cavity visualization. Orthogonal slices in the $x-y$ plane are also analyzed to study the cavity distribution along the tensile direction. The capability to visualize cavities inside the samples provides more information of the internal creep damage. Cavity morphology shows elongated shapes aligned along the rolling direction (coinciding with the tensile direction), which is consistent with the observation in the 2-D SEM images. The number of cavities on the orthogonal planes reduces progressively with increasing distance from the central neck plane, as shown in Fig. 12c. 


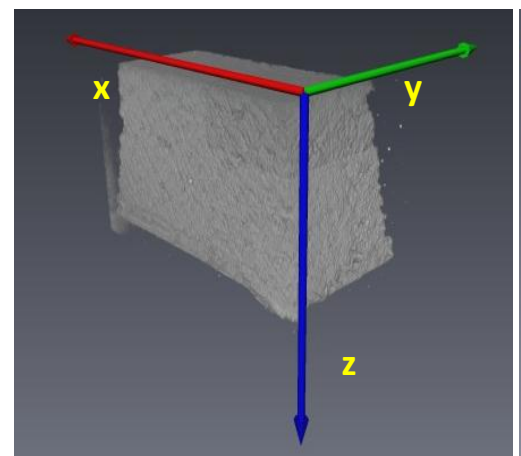

(a)

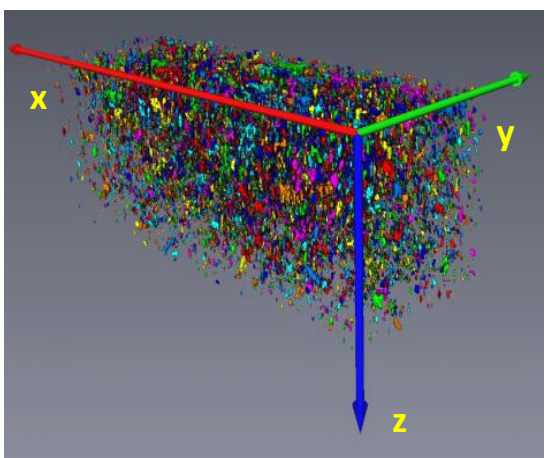

(b)

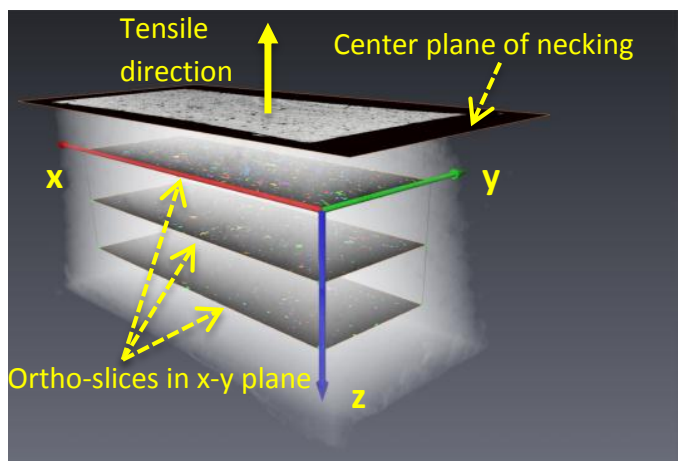

(c)

Fig. 12 15MPa, 133\% strain (a) Avizo 3D reconstructed sample, (b) 3-D cavity visualization, (c) ortho-slices along tensile direction

\subsection{Residual Material Strength}

As discussed above, the stress-induced damages on aluminum alloy are manifested as cavitation development and dynamic microstructure evolution. However, the impact of microstructural damage on post-fire material response still lacks full understanding. To evaluate material's post-fire performance, residual mechanical properties of damaged samples were determined from post-fire uniaxial tensile tests under ambient conditions. The residual $0.2 \%$ offset yield strength and ultimate strength for damaged samples are calculated based on deformed cross sectional area after creep tests.

To isolate the impact of stress-induced damage from thermally-induced damage, both unstressed thermal exposure and strained samples are tested to compare their post-fire residual mechanical properties. Material strength degradation during unstressed thermal exposure is primarily caused by static grain recrystallization during heating. As shown in Fig. 13, material heated to $400^{\circ} \mathrm{C}$ results in a decrease in residual yield and ultimate strengths of $160 \mathrm{MPa}$ and $50 \mathrm{MPa}$, respectively. As soak time increases for unstressed samples, no further decrease in residual strength is observed, which is due to the fact that static recrystallization is completed by the time material reaches $400^{\circ} \mathrm{C}$. Compared to unstressed samples exposed at $400^{\circ} \mathrm{C}$, residual material strengths of $400^{\circ} \mathrm{C}, 17 \mathrm{MPa}$ strained samples show similar yield strength value at low strain levels $(25 \%$ and $50 \%$ strain). However, residual yield strength increases by 30MPa over unstressed data at the high strain levels (100\% and $143 \%$ strain).

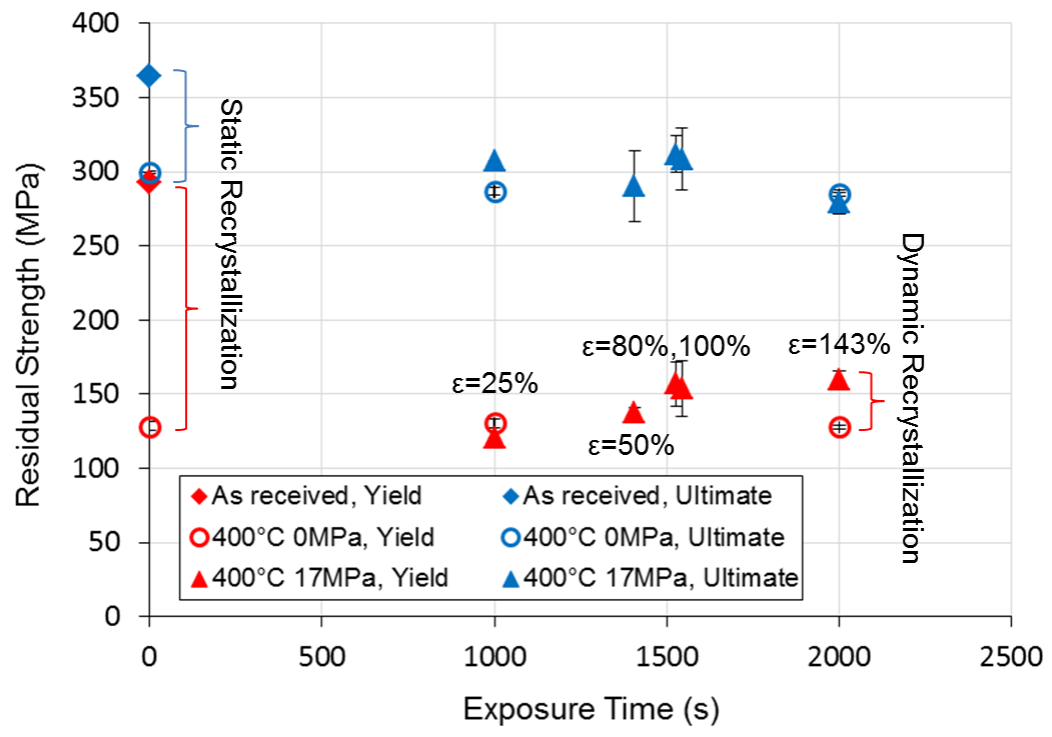

Fig. 13 Residual material strength based on deformed cross section area for solely thermal exposure and creep samples 
For samples treated at $400^{\circ} \mathrm{C}$ where microstructure has completely evolved by the time loading is applied, previous discussions reveal two competing mechanisms during the following creep deformation: cavitation which degrades the residual strength by introducing defects inside the material and grain elongation which strengthens the material by strain hardening. These two mechanisms are not significant at low creep strain microstructures; grains still maintain similar shape to as-received state and few cavities have appeared. Quantified data in Fig. 8a in section 4.2.1 and Fig. 10 in section 4.2.2 also verified minimal microstructural damage has taken place in the low strain samples. Thus almost no change in strength over the unstressed sample was measured at low strained samples of $25 \%$ and $50 \%$. However, effects of these two mechanisms are enhanced with an increase in creep strain. At high strain levels (100\% and 143\%) where cavitation is significant and grains become highly elongated, the two effects counteract each other and result in only a small difference in the yield strength.

The resulting increase in strength is attributed to the substructure strengthening mechanism associated with grain elongation at high stain levels. Previous discussion in section 4.2.2 reveals significant grain elongation in Fig. 10 with the increase of plastic strain: grain aspect ratio increases from 0.91 at the solely thermal exposure state to 7.5 at the highly strained state. During this process dynamic recovery takes place and dislocations are rearranged in the deformed grains. TEM inspection provides more evidence for the dislocation rearrangement associated with grain elongation. Microstructures which were solely heated to $400^{\circ} \mathrm{C}$ and strained to low and high plastic strain were examined. 50\% strain was selected to represent the low strain level with few damage, while $150 \%$ strain (slightly higher but close enough to $143 \%$ strain in above discussion) was chose to investigate the just-before-rupture state with significant grain elongation. As shown in Fig. 14, subgrain structures are clearly identified in TEM microstructures; dislocations at different configuration are compared for three states. Some layered structures shown in all three states are caused by the uneven thickness effect during TEM observation.

Fig. 14a displays the TEM microstructure solely heated to $400^{\circ} \mathrm{C}$, in which the primary feature is that dislocations are randomly distributed in subgrains and around precipitates. These randomly distributed dislocations are mark as ' $A$ ' configuration. The zoom in view of disorganized 'A' dislocation configuration is shown in Fig. 14b. After mechanical load is applied, dynamic recovery occurs simultaneously with the increase of material deformation. During transient both dislocation generation and annihilation rate increase until they reach a balance [33]. With increasing plastic deformation, dislocations will be annihilated and combined in low-energy array, which is marked as ' $\mathrm{B}$ ' configuration. Fig. 14c displays the ' $\mathrm{B}$ ' configuration formation in the 50\% strained microstructure. The zoom in view in Fig. 14d gives a clear view of dislocations piling up into an array at low strain state. However, only a few low-energy dislocation arrays are detected at low strained microstructure. With the accumulation of creep strain, more dislocation arrays are detected in the highly strained microstructure, as shown in Fig. 14e. Many of these dislocation arrays are observed to lap over and generate low angle dislocation cell walls inside subgrains, this type of configuration is marked as ' $\mathrm{C}$ '. Fig. $14 \mathrm{f}$ displays a region of ' $\mathrm{C}$ ' configuration in which dislocation cell wall is forming from dislocations lapping over in an array. The formation of dislocation walls from low-energy arrays results in the development of smaller subdomains and refinement of dislocation substructures. The substructure refinement during the transformation from ' $\mathrm{A}$ ' to ' $\mathrm{C}$ ' dislocation configuration will cause an increase in material strength based on the Hall-Petch model [22].

In addition to substructure refinement during dynamic recovery, dynamic recrystallization also takes place to promote dislocation displacement in highly strained grains. As shown in Fig. 9f, plenty of small recrystallized grains nucleate at the original grain boundaries of elongated grains to cause a reduce in average grain size. The grain refinement during dynamic recrystallization also contributes to the residual mechanical properties of aluminum alloy. The strength increments due to both grain boundary strengthening and substructures strengthening are both accounted in Hall-Petch model [22]. Thus, the increase in yield strength during grain elongation in Fig. 13 can be attributed to the substructure strengthening and dynamic recrystallization at high stain levels. 


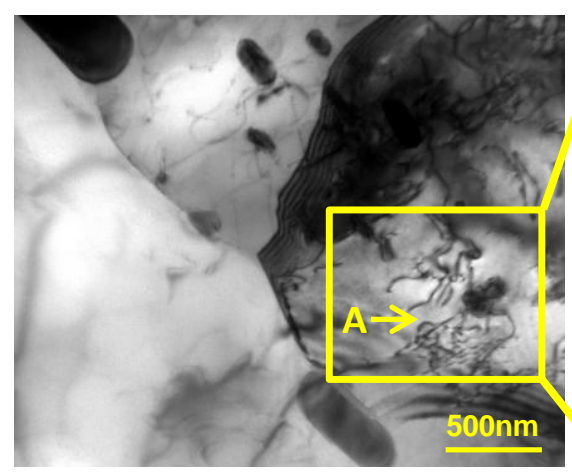

(a)

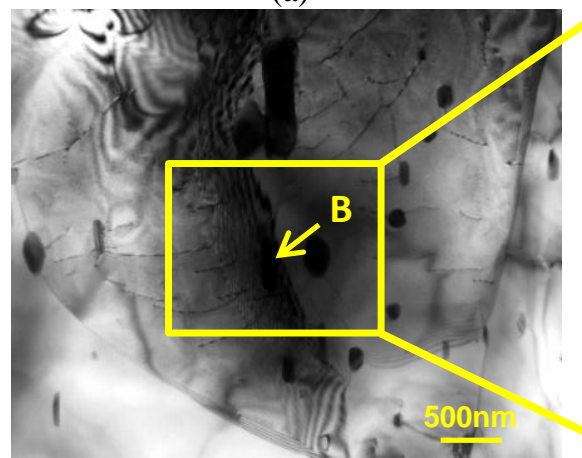

(c)

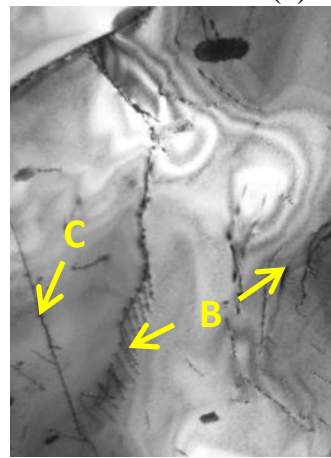

(e)

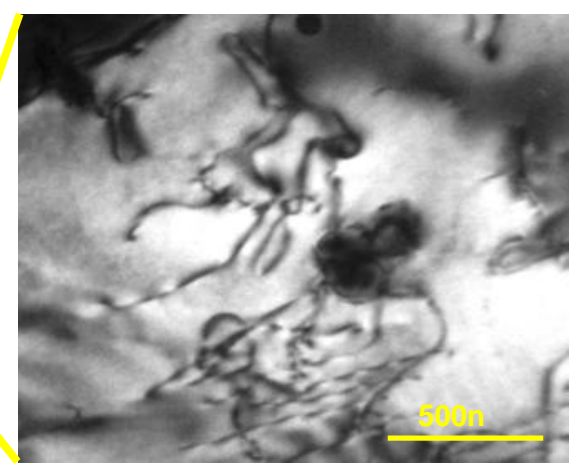

(b)

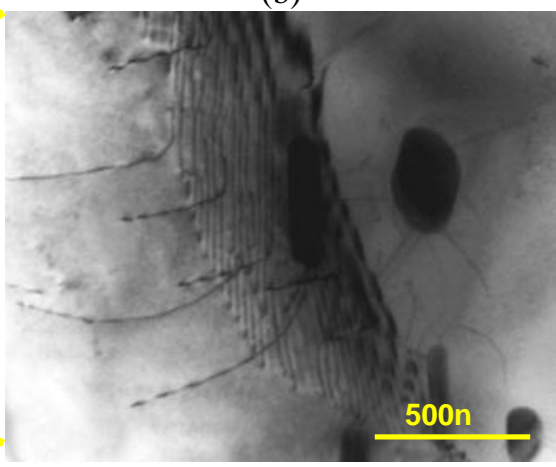

(d)

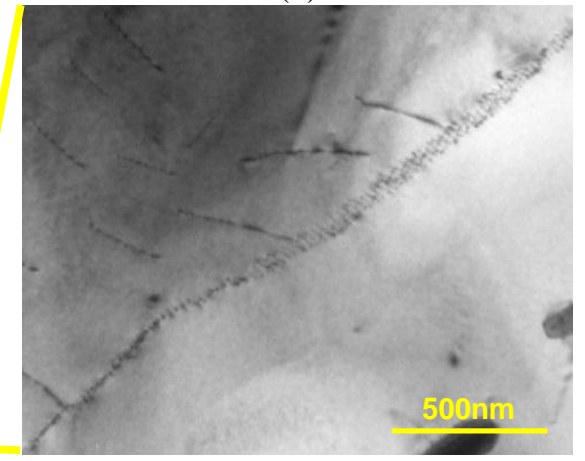

(f)

Fig. 14 TEM microstructures of (a) $400^{\circ} \mathrm{C}, 0 \%$ strain, (b) A configuration: randomly distributed dislocations

(c) $400^{\circ} \mathrm{C}, 17 \mathrm{MPa}, 50 \%$ strain, (d) B configuration: low energy dislocation array

(e) $400^{\circ} \mathrm{C}, 17 \mathrm{MPa}, 150 \%$ strain, (f) $\mathrm{C}$ configuration: low angle dislocation cell wall

\section{Conclusion}

This work examined the damage evolution in both unstressed thermal exposure and strained $\mathrm{Al} 15083$ samples heated to $400^{\circ} \mathrm{C}$. Two-dimensional microscopy and three-dimensional X-ray tomography were applied to study the external and internal stressinduced damage.

Cavitation and grain structure evolution at various creep strain levels were analyzed to study the damage mechanism at $400^{\circ} \mathrm{C}$ exposure. Stress concentration promotes cavity formation on the grain boundaries while creep plasticity dominates the subsequent cavity growth. Cavity morphology changes from a small spherical shape at low strain level to a large crack-like cylindrical shaped cavity orientated along the rolling/tensile direction at higher strain. Cavity volume fractions are quantified and exhibit an increase as a function of creep strain. Simultaneously, dynamic microstructural evolution causes grains to evolve from an equiaxed grain structure to a textured elongated grain structure at high strains. Grain aspect ratio increases significantly with the accumulation of creep strain. Small new grains nucleate in the locations where the serrated grain boundaries come into contact. 
The impact of stress-induced damage on the post-fire residual strength is minimal at the low strain levels due to the small amount of microstructural change. However, both cavitation and grain elongation are enhanced with the increase of creep strain. Dynamic recovery and dynamic recrystallization occurs associated with grain elongation to cause substructure refinement and average grain size reduction. The reduction in strength due to cavitation is counteracted by the substructure strengthening and dynamic recrystallization in highly strained microstructure. Thus the competing process between cavitation and grain elongation caused an increase in strength over unstressed samples despite significant cavitation at high strain levels.

\section{Acknowledgements}

The authors would like to acknowledge the Office of Naval Research for funding this work under Grant N00014-11-1-0875, scientific monitor Dr. William Mullins.

\section{Reference}

[1] F.A. Leckie, D.R. Hayhurst, Proc. R. Soc. Lond. A, (1974) 323-347.

[2] J. Lin, Y. LIU, T.A. DEAN, International Journal of Damage Mechanics, 14 (2005) 299-319.

[3] G. Hénaff, G. Odemer, B. Journet, Aluminium Alloys, Theory and Applications, (2011) 259-282.

[4] H.E. Evans, London : Elsevier Applied Science, (1984).

[5] M.E. Kassner, T.A. Hayes, Int J Plasticity, 19 (2003) 1715-1748.

[6] J.N. Greenwood, D.R. Miller, J.W. Suiter, Acta Metallurgica, 2 (1954) 250-258.

[7] M.H. Yoo, H. Trinkaus, Acta Metallurgica, 34 (1986) 2381-2390.

[8] H. Trinkaus, M.H. Yoo, Philos Mag A, 55 (1987) 269-289.

[9] P.M. Anderson, P.G. Shewmon, Mech Mater, 32 (2000) 175-191.

[10] D. Hulla, D.E. Rimmera, Philosophical Magazine, 4 (1959) 673-687.

[11] J.W. Hancock, Met Sci, 10 (1976) 319-325.

[12] R. Raj, M.F. Ashby, Acta Metallurgica, 23 (1975) 653-666.

[13] I.W. Chen, Scripta Metall Mater, 17 (1983) 17-22.

[14] K. Kannan, C.H. Hamilton, Scripta Mater, 38 (1998) 299-305.

[15] N. Chandra, Z. Chen, Materials Research Society, 601 (2000) 235-246.

[16] I.C. Hsiao, J.C. Huang, Metallurgical and Materials Transction A, 33A (2002) 1373-1384.

[17] F.M. R. Kaibyshev, D.R. Lesuer, T.G. Nieh, Materials Science and Engineering, A342 (2003) 169-177.

[18] A.C.F. Cocks, M.F. Ashby, Met Sci, 16 (1982) 465-474.

[19] E.O. Hall, Proceedings of the Physical Society. Section B, 64 (1951) 747.

[20] H.J. Bunge, F. Wagner, P.I. Welch, P. Vanhoutte, J Phys Lett-Paris, 46 (1985) 1109-1113.

[21] S. Sun, V. Sundararaghavan, Acta Mater, 60 (2012) 5233-5244.

[22] E.L. Huskins, B. Cao, K.T. Ramesh, Materials Science and Engineering: A, 527 (2010) 1292-1298.

[23] J.Y. Uan, H.F. Cheng, Materials Transactions, 48 (2007) 178-183.

[24] R.D. Matulich, M.S. thesis, Virginia Tech, (2011).

[25] M. Dierick, B. Masschaele, L.V. Hoorebeke, Measurement Science and Technology, 15 (2004) 1366-1370.

[26] K.H. Tsutomu Tanaka, Materials Transactions, 45 (2004) 2547-2551.

[27] M.K. Rao, B.P. Kashyap, A.K. Mukherjee, Pergamon Press, Oxford, (1984) 2311-2317.

[28] K. Kannan, C.H. Hamilton, Scripta Mater, 38 (1997) 299-305.

[29] A.C.F. Cocks, M.F. Ashby, Met Sci, 14 (1980).

[30] B. Budiansky, J.W. Hutchinson, S. Slutsky, Mechanics of Solids, The Rodney Hill 60th Anniversary Volume (1982).

[31] E.V. Konopleva, H.J. Mcqueen, E. Evangelista, Mater Charact, 34 (1995) 251-264.

[32] S.H. Goods, W.D. Nix, Acta Metallurgica, 26 (1978) 753-758.

[33] H.J. Mcqueen, S. Spigarelli, M.E. Kassner, E. Evangelista, CRC Press (2011). 